\title{
A Salt-free and Water-saving Approach as a Green Alternative to Conventional Reactive Dyeing of Cotton
}

\author{
Yimin Wei \\ Jiangnan University \\ Zhe Jiang \\ Jiangnan University \\ Ning Zhang \\ Jiangnan University \\ Ping Wang \\ Jiangnan University \\ Yuanyuan Yu \\ Jiangnan University \\ Man Zhou \\ Jiangnan University
}

Qiang Wang ( $\square$ qiang_wang@163.com )

Jiangnan University https://orcid.org/0000-0002-8461-6552

\section{Research Article}

Keywords: Hydrophobic deep eutectic solvent system, salt-free, cotton fabrics, recyclable, clean dyeing

Posted Date: May 17th, 2021

DOl: https://doi.org/10.21203/rs.3.rs-494140/v1

License: (9) This work is licensed under a Creative Commons Attribution 4.0 International License.

Read Full License

Version of Record: A version of this preprint was published at Sustainable Chemistry and Pharmacy on December 1st, 2021. See the published version at https://doi.org/10.1016/j.scp.2021.100536. 


\section{A salt-free and water-saving approach as a green alternative to conventional reactive dyeing of cotton}

Yimin Wei, Zhe Jiang, Ning Zhang, Qiang Wang *, Ping Wang, Yuanyuan Yu, Man Zhou

\section{Corresponding Author: *Qiang Wang. E-mail: qiangwang@jiangnan.edu.cn}

Key Laboratory of Science and Technology of Eco-Textile, Ministry of Education, Jiangnan University, Wuxi, Jiangsu, 214122, China

\section{Abstract}

Aqueous reactive dyeing of cotton is considered to be environmentally unfriendly owing to its high consumption of water and salt as well as large discharge of wastewater. However, the industrial applications of current alternative dyeing technologies are difficult to realize due to different problems such as poor dyeing quality, environmental pollution and high cost. Herein, we developed a hydrophobic deep eutectic solvent (HDES) dyeing approach using natural thymol-menthol to overcome the problems caused by reactive dyeing of cotton. HDES dyeing approach could achieve excellent dyeing properties of cotton by optimizing water content, pick-up and concentration of alkali solution. Dye fixation kinetics of HDES dyeing system was studied based on Lagergren pseudo-first-order and pseudo-second-order dynamics models, compared with aqueous dyeing system. The cotton fabrics dyed in Thy-Men system exhibited excellent levelness, satisfactory colorfastness and color strength without addition of salt. Furthermore, HDES can be reused for 5 cycles of 
dyeing and the dyed fabrics displayed consistently high color strength values. This dyeing approach provides substantial reduction in wastewater emission and consumption of freshwater and chemicals, which has considerable potential for promoting cleaner production in the textile industry.

Keywords Hydrophobic deep eutectic solvent system; salt-free; cotton fabrics; recyclable; clean dyeing

\section{Introduction}

Cotton has been widely used because of outstanding characteristics, such as high air permeability, excellent softness, good comfort as well as being easy to dye (Wang et al. 2009). Reactive dyes have become the main dyes for cotton fibers due to their bright colors, complete chromatograms, satisfactory colorfastness and simple dyeing process (Shu et al. 2018). However, conventional aqueous reactive dyeing of cotton requires huge amounts of electrolyte, such as $\mathrm{NaCl}$ and $\mathrm{Na}_{2} \mathrm{SO}_{4}$, to suppress the electrostatic repulsion between fibers and dyes, thus promoting the adsorption of dyes (Suwanruji and Freeman 2010). In addition, a large amount of water and alkali are also consumed (Arivithamani and Giri Dev 2017). Reactive dyeing has become one of the major generators of wastewater in textile processing (Li et al. 2019). Such unrecyclable effluents are harmful to aquatic biota, humans and even the entire ecological environment (Ayadi et al. 2016; Vakili et al. 2014). With increasingly stringent environmental regulations, developing new dyeing technologies with the aim at decrease in water consumption and effluent emission is critical to the sustainable development of textile industry. Although various methods (Jiang et al. 2018; Libra et 
al. 2004; Xia et al. 2020) have been applied to treat contaminated effluent, the cost of treatment is high and the consumption of freshwater resources cannot be significantly reduced. Reduction in dyeing wastewater generation is the most effective method based on the "3Rs" principle, known as reduce, reuse and recycle (Mu et al. 2019c; Tam and Tam 2006).

Many researchers have realized the importance of developing a green approach for reactive dyeing of cotton. Though supercritical carbon dioxide has the potential to replace water (Fernandez Cid et al. 2005; Long et al. 2014; Luo et al. 2018) nonpolar $\mathrm{ScCO}_{2}$ cannot effectively swell cotton characterized by high polarity, leading to low dye sorption and poor color build-up (Cid et al. 2007). Furthermore, the dyeing technology is expensive as high pressure is required, which greatly limits large-scale industrial application of $\mathrm{ScCO}_{2}$ dyeing. Cationic modification of cotton fiber is another approach to promote dye absorption and reduce salt consumption (Dong et al. 2020; Hauser and Tabba 2001; Ma et al. 2016). However, large amounts of cationic auxiliaries are required, causing additional wastewater pollution. Organic solvent dyeing has received considerable interest in recent decades. As single non-nucleophilic organic solvent cannot swell cotton effectively, polar-non-polar systems have been developed to reduce the hydrolysis of reactive dyes, including N,N-dimethylacetamide (DMAc)/dimethylcarbonate (DMC) (Chen et al. 2015), Ethyl octanoate (EO)/dimethyl sulfoxide (DMSO) (Zhao et al. 2018) and N,N-Dimethylformamide (DMF)/trichloroethylene (Chavan and Rao 1983). It is tough to achieve zero emission of organic solvents in the dyeing process, which is 
unfriendly to the environment. Besides, dye fixation is unsatisfactory in organic solvent dyeing system. Decamethylcyclopentasiloxane (D5)-water system (Fu et al. 2015; Pei et al. 2021), oil-water system (Liu et al. 2019; Mu et al. 2019a; Mu et al. 2019b) and ethanol-water system (Wang et al. 2020; Xia et al. 2021; Xia et al. 2018) also have been investigated to realize the aim of clean dyeing since the 1950s. The dye and D5 could form the dyeing system by ball-milling and reversed micellar, which could realize cotton dyeing without salt. However, levelness of cotton dyeing in ball-milling system was not satisfactory because of the rapid dyeing of dyes. The dye fixation was low in reversed micellar dyeing system. Moreover, D5 is not biodegradable and could cause safety and environmental concerns (Sparham et al. 2008), which hinders the industrialization of the technology. In summary, current novel reactive dyeing systems cannot replace the water system completely in commercial use. Therefore, developing a green, safe and recyclable non-aqueous dyeing technology is the key to solve the problems of reactive dyeing.

Deep eutectic solvents (DESs), known as a new generation of green solvents (Cao et al. 2017), have attracted considerable attention in recent decades. DESs can substitute for organic solvents as they could obey the Green Chemistry criteria (Anastas and Warner 1998). DESs can be obtained by mixing hydrogen bond donors and hydrogen bond acceptors in a certain proportion. The components of DESs are connected through hydrogen bonding (Durand et al. 2016). The majority of DESs researched are hydrophilic, this greatly restricts their practical application because of the miscibility with water. Many researchers have turned their attention to 
hydrophobic DESs. HDESs have been used to remove metal ions, pesticides in water (Florindo et al. 2014; van Osch et al. 2016) and extract leaf components (Cao et al. 2017), but as far as we know, there is no relevant report in the field of dyeing application. Hydrophobic deep eutectic solvents (HDES) composed of natural components are considered to be more environmentally friendly (Paiva et al. 2014). Menthol (Men), a terpene, can be extracted from Mentha species and is often used to form a hydrophobic eutectic solvent system. Since the fixation of reactive dyes requires alkaline conditions, acidic substances are unacceptable in the solvent system. According to the report, thymol (Thy), a natural component, can form HDES with menthol at a certain temperature (van Osch et al. 2019). Thy-Men has excellent features of nontoxic, non-flammable, biodegradability and biocompatible, making it possible to replace water as an environmental protection dyeing medium.

In our present work, thymol-menthol was used as a medium for cotton dyeing. The Thy-Men dyeing system can decrease the consumption of salt and freshwater, as well as minimize the discharge of the dyeing pollutants. The dyeing procedures are shown in Scheme 1. Water was used to swell the cotton and dissolve reactive dyes, blended with thymol-menthol at high speed to form a water-in-oil system. Cotton fabrics dyed by this approach have quality comparable to those by traditional way in the absence of electrolytes. Compared to dyeing systems reported previously, Thy-Men system can realize recyclable dyeing without any complicated steps. In summary, the green and sustainable dyeing process makes Thy-Men become a promising substitute for water. 


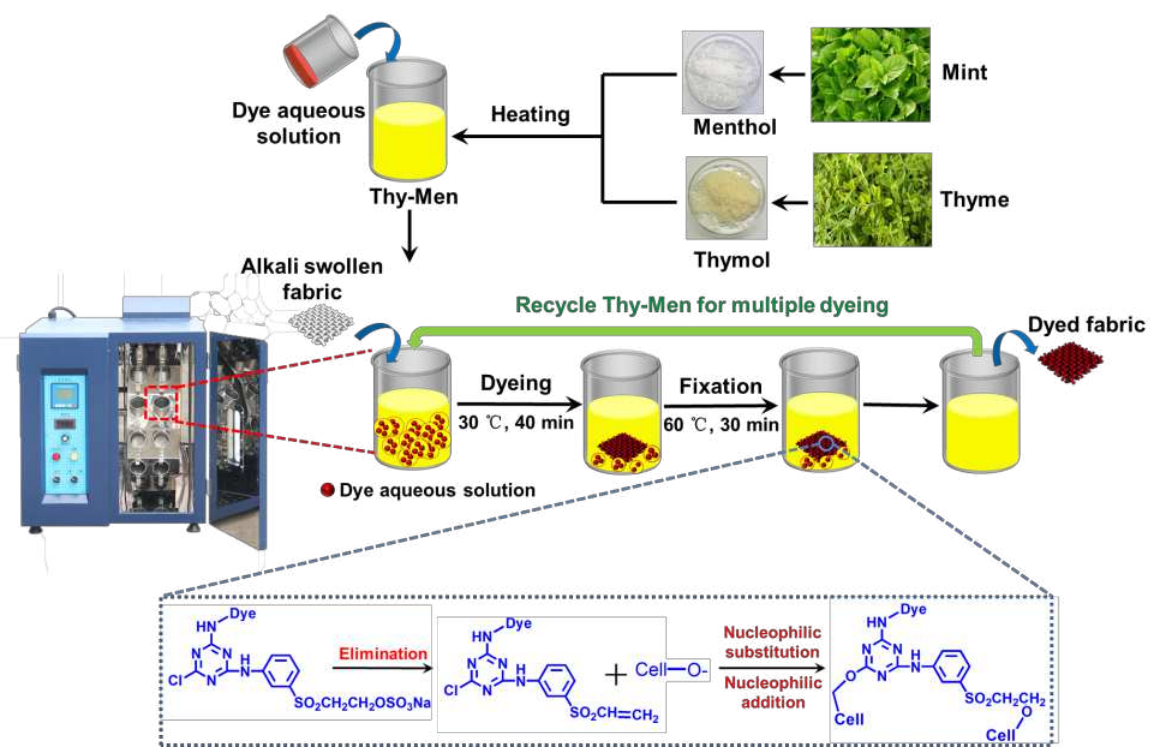

Scheme 1 Procedure for salt-free reactive dyeing of cotton fabrics in Thy-Men system

\section{Experimental Section}

\section{Materials}

Menthol was ordered from Guangzhou Daily Chemical Co., Ltd. Thymol was bought from Aladdin Chemical Reagent. Sodium sulfate $\left(\mathrm{Na}_{2} \mathrm{SO}_{4}\right)$, anhydrous sodium carbonate $\left(\mathrm{Na}_{2} \mathrm{CO}_{3}\right)$ and sulfuric acid were obtained from Sinopharm Chemical Reagent Co., Ltd. of China. C.I. Reactive Red 195 (RR195), Reactive Blue 194 (RB194) and Reactive Yellow 145 (RY145) were provided by McLin Biochemical Technology Co., Ltd, Shanghai. Fig. 1 provides the chemical structures of different reactive dyes. Desized, scoured and bleached plain weave cotton fabrics were supplied by the Shandong Ruyi Group Co., Ltd., China. 
(a)

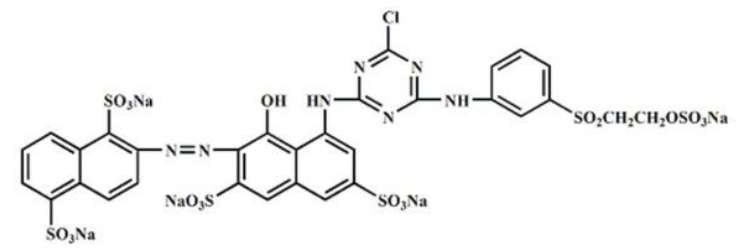

(b)

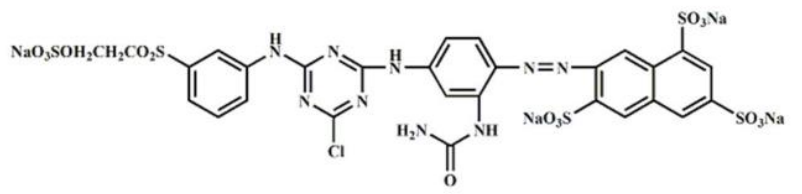

(c)

Fig. 1 Chemical structure of (a) C. I. Reactive Red 195; (b) C. I. Reactive Yellow 145; (c) C. I. Reactive Blue 194

\section{Preparation of the hydrophobic deep eutectic solvent}

The HDES was synthesized according to literature procedures (van Osch et al. 2019). Menthol (1 mol) and Thymol ( $1 \mathrm{~mol})$ were heated at $50{ }^{\circ} \mathrm{C}$. The HDES was obtained when the liquid became homogeneous.

\section{Dyeing process}

First, the cotton fabrics were dipped in an aqueous sodium carbonate solution at room temperature for an hour and then squeezed under different pressures to obtain a proper liquor pick-up before dyeing. The dye bath was achieved by mixing Thy-Men and water with dissolved dyes at high speed for 2 minutes. The weight of water content in the dye bath was $60-140 \%$ o.w.f (on weight of fabric). Subsequently, the alkali-treated fabrics were immersed in the prepared dye bath. The dyeing bath began with $30^{\circ} \mathrm{C}$ for 40 min to transfer dye to fabric surface completely and then warmed to $60^{\circ} \mathrm{C}$ for $30 \mathrm{~min}$ to realize the dye fixation of the cotton. The entire dyeing process 
was carried out on the infrared dyeing machine. The liquor-to-goods ratio is 20:1 and dye concentration was $2 \%$ o.w.f. Water content, alkali concentration, alkali solution pick-up were optimized according to the dyeing effect. For reuse of the HDES, the spent bath after one cycle of dyeing was collected. Thy-Men without any dye was taken out to reuse for the next dyeing cycle after removing a small amount of the remaining dye aqueous solution.

As a control, conventional aqueous dyeing was conducted. The concentrations of the dye and the fabric-to-liquor ratio are the same as thy-men dyeing process. Specifically, cotton fabrics dyed in bath at $60{ }^{\circ} \mathrm{C}$ for $100 \mathrm{~min} .40 \mathrm{~g} / \mathrm{L} \mathrm{Na}_{2} \mathrm{SO}_{4}$ and 20 $\mathrm{g} / \mathrm{L} \mathrm{Na}_{2} \mathrm{CO}_{3}$ was gradually added into the bath.

After dyeing, cotton fabrics were washed with water for $10 \mathrm{~min}$ and then soaped in a solution at $95{ }^{\circ} \mathrm{C}$ for $10 \mathrm{~min}$ (liquor ratio $=30: 1$ ), which was composed of $\mathrm{Na}_{2} \mathrm{CO}_{3}$ and AATCC standard detergent ( $2 \mathrm{~g} / \mathrm{L}$ ). Subsequently, cotton fabrics were rinsed thoroughly using hot water and cold water before being dried.

\section{Measurements}

Nuclear Magnetic Resonance

Carbon $\left({ }^{13} \mathrm{C}\right)$ nuclear magnetic resonance was conducted to confirm the formation of the hydrophobic eutectic solvent. The experiments were carried out on a Bruker 400 spectrometer.

Color strength and dyeing levelness measurement The color strength $(\mathrm{K} / \mathrm{S})$ and $\mathrm{L} * \mathrm{a}^{*} \mathrm{~b} *$ values of dyed fabrics were measured with the Datacolor 850 spectrophotometer. The K/S values were determined according to 
the Kubelka-Munk equation, as shown in eqn (1):

$$
K / S=\left(1-R^{2}\right) / 2 R
$$

CIE $\mathrm{L}$, $\mathrm{a}$ and $\mathrm{b}$ from 10 points on one sample were measured to evaluate levelness. The color difference $(\Delta \mathrm{E})$ was calculated by the equation according to the values of CIE LAB. (Eq.2).

$$
\Delta \mathrm{E}=\left[\left(\Delta \mathrm{L}^{2}\right)+\left(\Delta \mathrm{a}^{2}\right)+\left(\Delta \mathrm{b}^{2}\right)\right]
$$

$\Delta \mathrm{L}, \Delta \mathrm{a}$ and $\Delta \mathrm{b}$ are the differences in the color parameters of the dyed samples. Largest $\Delta \mathrm{E}$ less than 1 indicates good levelness.

Colorfastness of dyed cotton fabrics

The fastness to crocking was performed according to ISO 105-X12 using a YB571 rubbing fastness tester. The washing fastness was carried out according to ISO 105-C06 using an SW-12AC washing colorfastness tester. Color degradation was evaluated by visual assessment based on the grey scale in an appropriate light cabin.

Dye fixation

Dye fixation was measured according to a procedure previously reported by Kissa (Kissa 1971). Cutting the dyed samples into small pieces and then heat at $105^{\circ} \mathrm{C}$ for 2 hours. The dye is stripped from the fabric with $70 \%$ sulfuric acid after the fabric is cooled and weighed accurately. The amount of the dye in cotton was measured using a UV-1800 spectrophotometer (Shimadzu Corporation, Japan). The calibration curve had $\mathrm{R}^{2}>0.999$.

Dyed fabrics cross-section Cross-sections of the dyed fabrics were prepared as follows: Several yarns were 
extracted from the dyed fabrics and then frozen at low temperature with an embedding agent. Subsequently, the frozen samples were microtomed on the CM1950 slicer to 20 mm thickness and mounted on a slide glass. Cross-sectional images of the samples were obtained using a VHX-1000C ultra depth of field microscope.

Dye fixation kinetics

The fixation of RR195 onto fabric was carried out at $60{ }^{\circ} \mathrm{C}$ for $5-120$ min to ensure the balance of fixation. Color stripping was used to obtain the dye concentration on the fabric $[D]_{f}$ at time t. The fixation kinetics was illustrated with the pseudo-first-order and pseudo-second-order models.

$\mathrm{X}$-ray diffraction (XRD) and tensile properties analysis

Crystallinity of cotton fabrics was measured. The scanning was carried out in a $2 \Theta$ range of $5^{\circ}-60^{\circ}$ at a rate of $4 \% \mathrm{~min}$. Tensile properties of cotton fabrics were determined according to ISO standard 13934-1 2013. The samples were tested in their warp direction.

\section{Results and discussion}

\section{${ }^{13}$ C NMR of the HDES}

As shown in Fig. 2, the peaks in Thy-Men corresponded to the peaks of the original components, and no new peaks appeared, which confirmed that there was no chemical reaction between the components, but only a physical change process. As shown in insets, menthol and thymol are both solids and they become liquid mixture after heating, thus proving the formation of the HDES. 
(a)

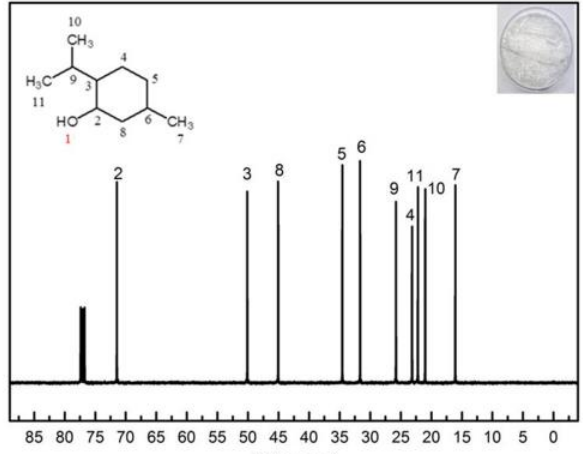

(c)

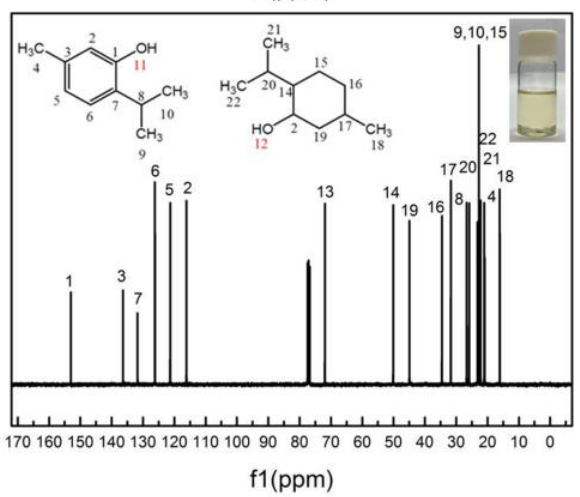

(b)

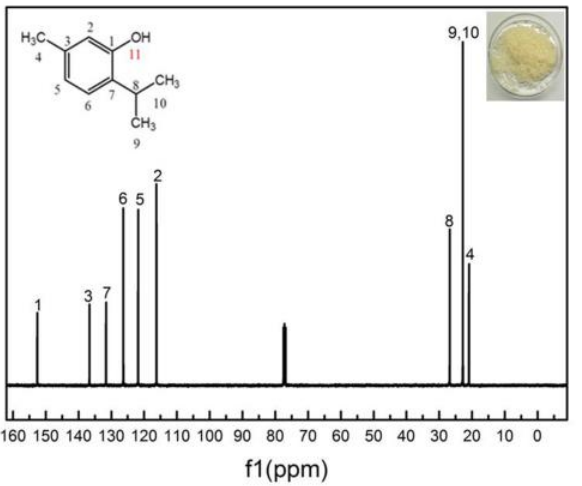

206

207

208

209

210

211 menthol, thymol and Thy: Men, respectively

Fig. $2{ }^{13} \mathrm{C}$ NMR of (a) menthol (b) thymol (c) Thy: Men (1:1). Insets show photos of

\section{Effect of water content of dyeing system, pick-up and concentration of alkali}

\section{solution on dyeing}

Dyeing with RR195 (2\% o.w.f) was conducted to examine influence of different water content on dyeing. The results are demonstrated in Fig. 3a, when the water content was less than $100 \%$ o.w.f, the $\Delta \mathrm{E}$ values were much higher than 1. It indicates extremely poor evenness. As the droplets containing dyes could not contact with the fabrics sufficiently and evenly, the fabrics had "white space", resulting in poor levelness and a low final $\mathrm{K} / \mathrm{S}$ value. As the water content increased to $100 \%$ o.w.f, there is enough water in the dyeing system to obtain satisfactory levelness of the dyed fabrics. Further increase of water content had a negative effect on dyeing quality. Taking both color strength and levelness into 
consideration, the water content of $100 \%$ o.w.f was determined as the best condition.

In addition to water content of dyeing system, pick-up and concentration of 

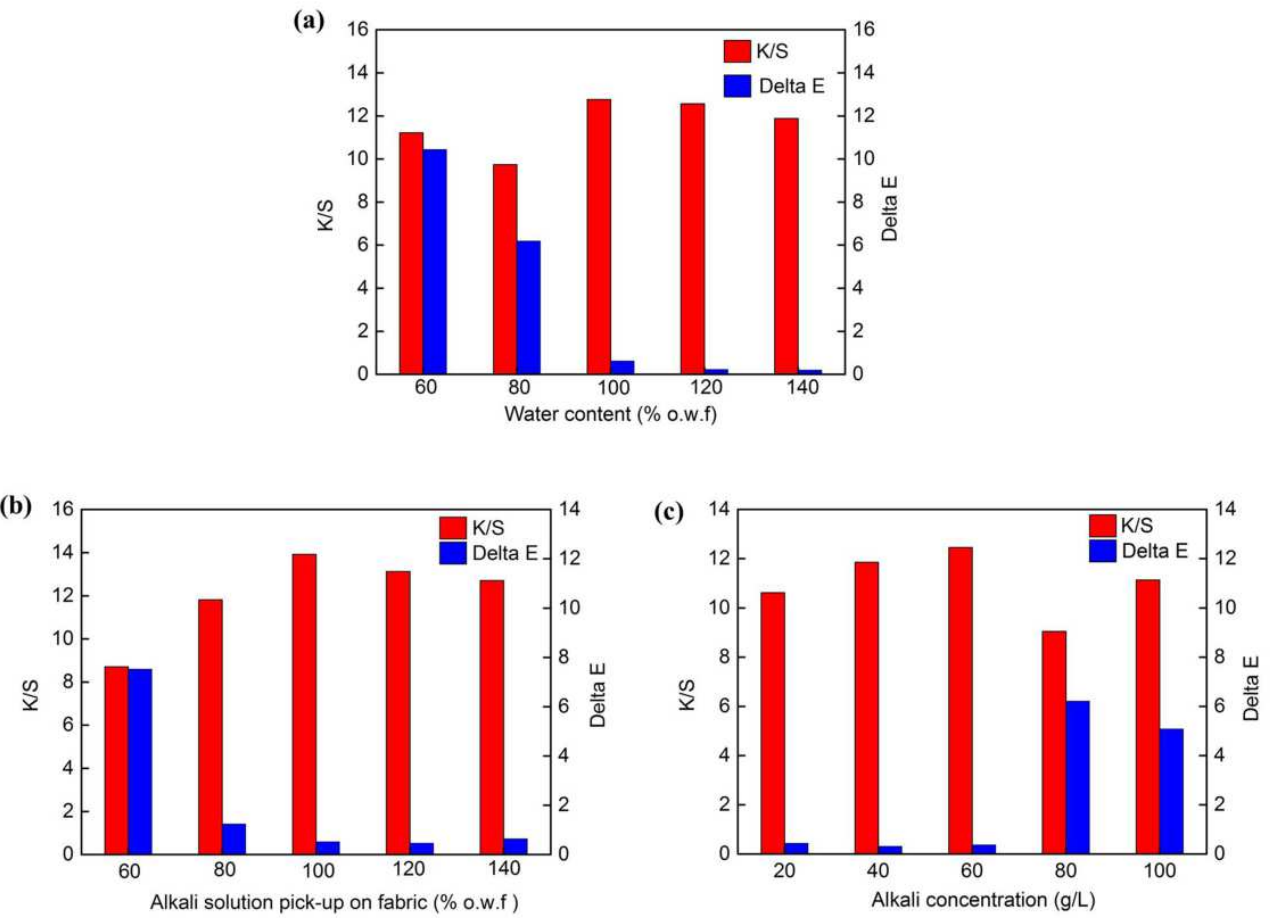

Fig. 3 Effect of (a) water content (b) pick-up and (c) concentration of alkali solution on color strength $(\mathrm{K} / \mathrm{S})$ and levelness $(\Delta \mathrm{E})$

\section{Comparison of aqueous and Thy-Men dyeing with different dyes}

To evaluate the feasibility of Thy-Men dyeing system, dyeing was conducted with various reactive dyes. Besides, conventional dyeing using these reactive dyes was carried out for comparison. As indicated by Table 1, all the investigated dyes showed a satisfactory total fixation in the Thy-Men dyeing system. Compared with aqueous dyeing, cotton fabrics dyed in the Thy-Men system could also achieved high $\mathrm{K} / \mathrm{S}$ values without addition of salt. The mean colour difference values $(\Delta \mathrm{E})$ of the salt-free dyed fabrics were less than 1, indicating the fabrics dyed in the Thy-Men system can obtain a good level dyeing property. The figures of dyed samples in Fig. 4 also confirmed the above results. The dyeing quality of fabrics dyed in Thy-Men system was the same as that in aqueous system. 

systems

\begin{tabular}{|c|c|c|c|c|c|c|c|}
\hline Dyes ( $2 \%$ o.w.f) & Dyeing system & $\mathrm{K} / \mathrm{S}$ & $\Delta \mathrm{E}$ & $\mathrm{L}^{*}$ & $a^{*}$ & $b^{*}$ & $\mathrm{~T}(\%)$ \\
\hline \multirow{2}{*}{ RR195 } & Water & 11.852 & 0.13 & 45.62 & 61.22 & 0.96 & 68.37 \\
\hline & Thy-Men & 11.642 & 0.14 & 45.49 & 60.75 & 0.53 & 75.03 \\
\hline \multirow{2}{*}{ RY145 } & Water & 11.009 & 0.55 & 73.40 & 30.74 & 79.18 & 74.30 \\
\hline & Thy-Men & 10.632 & 0.31 & 73.60 & 31.07 & 78.97 & 68.95 \\
\hline \multirow[b]{2}{*}{ RB194 } & Water & 18.901 & 0.07 & 26.24 & -3.58 & -18.88 & 75.41 \\
\hline & Thy-Men & 18.561 & 0.03 & 26.90 & -3.86 & -19.25 & 81.28 \\
\hline
\end{tabular}

(a)

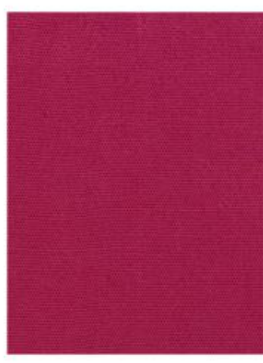

(c)

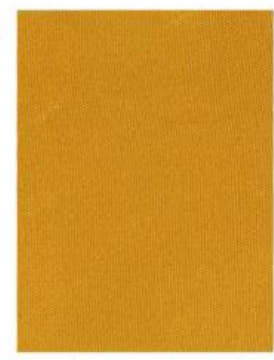

(b)

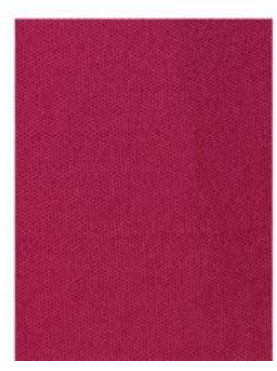

(d)

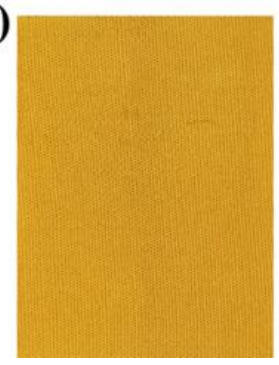

(e)

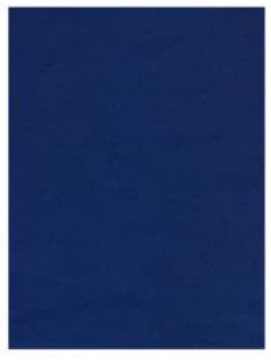

(f)

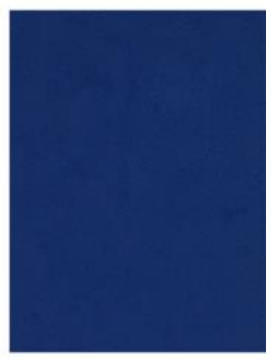

Fig. 4 Photos of fabrics dyed with: (a) RR195 (c) RY145 (e) RB194 in aqueous

(d) RY145 (f) RB194 in the Thy-Men system

The cross-sections of the fabrics dyed with various dyes were obtained to 259 evaluate the distribution of dyes in the fibers. As the images shown in Fig. 5, the cross-sections of samples dyed in the Thy-Men system were coloured evenly without white core phenomena. The colour depth of cross sections of cotton fabrics was also 

obtained in the Thy-Men system.
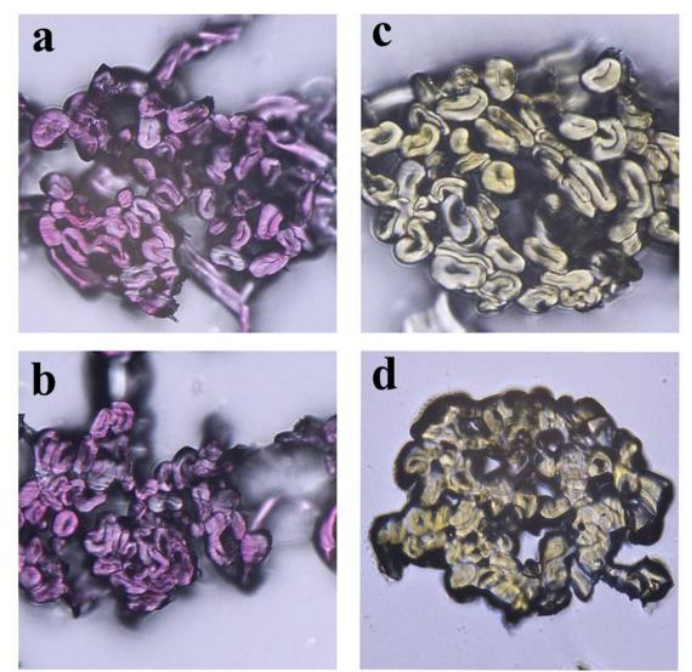
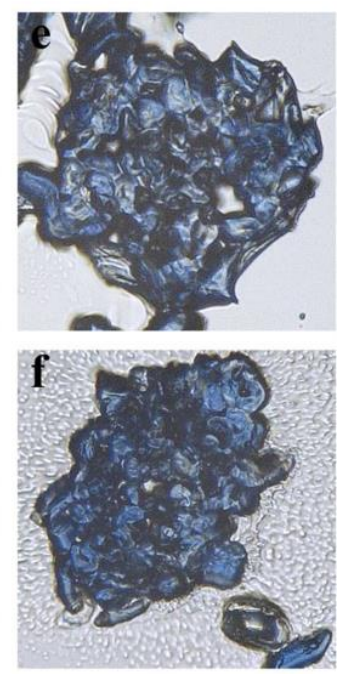

Fig. 5 Cross-sectional images of cotton yarns dyed with: (a) RR195 (c) RY145 (e)

RB194 in aqueous system; (b) RR195 (d) RY145 (f) RB194 in the Thy-Men system

Fig. 6 shows the K/S and the reflectance curves of dyed cotton fabrics. The K/S curves of the fabrics dyed in the Thy-Men system have the same maximum absorption wavelength as those dyed in aqueous system. The results are consistent with the $\mathrm{L}^{*}$, $a^{*}$ and $b^{*}$ values of the samples shown in Table 1 , suggesting that the tone and chromaticity zone of fabrics dyed in Thy-Men system were not changed. Furthermore, the reflectance value reflects the color depth of the fabric. As seen in Fig. 6, the fabrics dyed by two methods with various reactive dyes have the same reflectance values, which means the same color depth. 
(a)

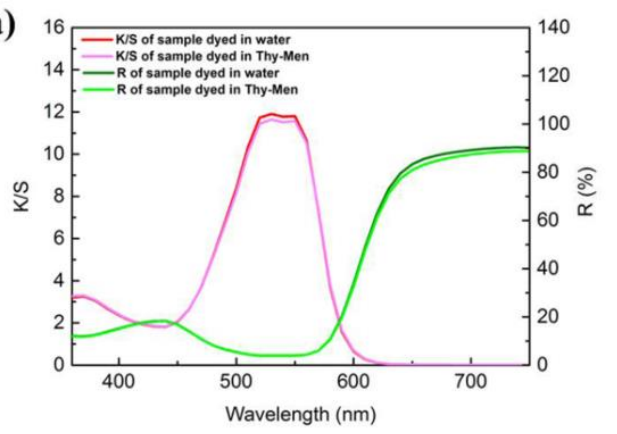

(c)

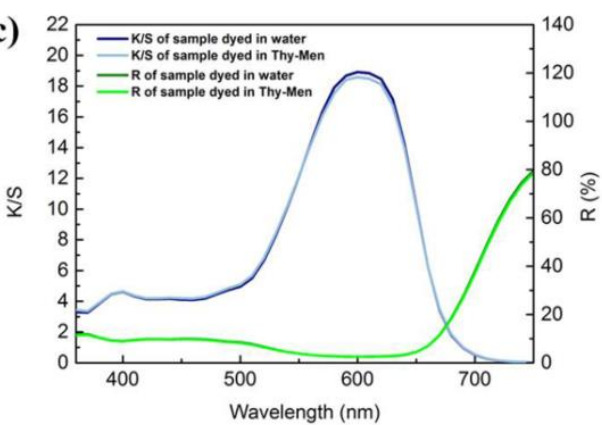

(b)

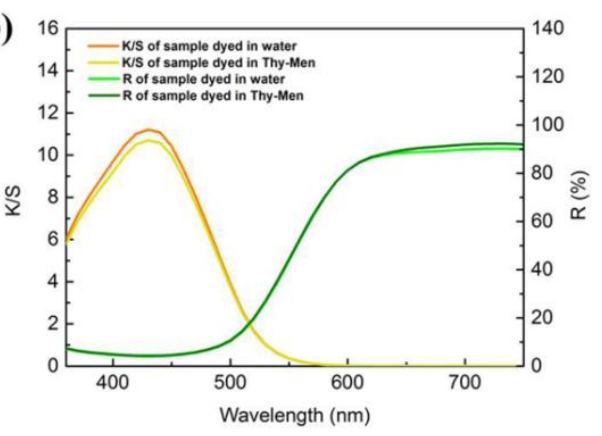

276

277

Fig. 6 Reflectance (R) and K/S curves of fabrics dyed with: (a) RR195; (b) RY145;

(c) RB194 in the Thy-Men system

The colorfastness properties of Thy-Men dyed fabrics and conventional dyed fabrics were compared in Table 2. Samples dyed in the Thy-Men system exhibited excellent colorfastness to crocking and washing comparable to samples dyed in aqueous system. These results demonstrate that quality of Thy-Men dyed fabrics fully meets the actual use requirements. In view of environmental protection and excellent dyeing effect, this dyeing system has the potential to replace the traditional dyeing system. 


\begin{tabular}{|c|c|c|c|c|c|c|c|c|c|c|}
\hline \multirow{3}{*}{ Dyes } & \multicolumn{3}{|c|}{ Rubbing } & \multicolumn{7}{|c|}{ Washing fastness } \\
\hline & system & \multirow{2}{*}{ Dry } & \multirow{2}{*}{ Wet } & \multirow{2}{*}{$\begin{array}{l}\text { Color } \\
\text { change }\end{array}$} & \multicolumn{6}{|c|}{ Staining $^{\mathrm{a}}$} \\
\hline & & & & & $\mathrm{C}$ & $\mathrm{N}$ & $\mathrm{P}$ & $\mathrm{A}_{1}$ & W & $\mathrm{A}_{2}$ \\
\hline \multirow{2}{*}{ RR195 } & Water & 5 & $4-5$ & 5 & 5 & 5 & 5 & 5 & 5 & 5 \\
\hline & Thy-Men & 5 & $4-5$ & 5 & 5 & 5 & 5 & 5 & 5 & 5 \\
\hline \multirow{3}{*}{ RY145 } & Water & 5 & $4-5$ & 5 & 5 & 5 & 5 & 5 & 5 & 5 \\
\hline & & & & & & & & & & \\
\hline & Thy-Men & 5 & $4-5$ & 5 & 5 & 5 & 5 & 5 & 5 & 5 \\
\hline \multirow{3}{*}{ RB194 } & Water & 5 & $4-5$ & 5 & 5 & $4-5$ & 5 & 5 & 5 & 5 \\
\hline & & & & & & & & & & \\
\hline & Thy-Men & 5 & $4-5$ & 5 & 5 & $4-5$ & 5 & 5 & 5 & 5 \\
\hline
\end{tabular}

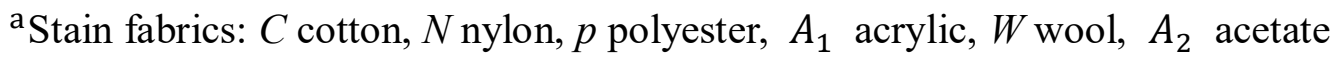

\section{Reusability of HDES for multiple cotton dyeing}

296 solubility, resulting in the discharge of dark waste dye bath with high salt content.

297 Chemical potential of dyes in Thy-Men system is high due to very limited solubility,

298 which makes it possible to dye the fabrics without the addition of salt. Thy-Men from

299 the spent bath was free of any dyes. Because a small amount of water was thrown out 300 of the fabric surface during the dyeing process, the bottom layer of the waste bath is a 
301

302

303

304

305

306

307

trace of dye aqueous solution. After a simple separation of the waste bath, Thy-Men can be recovered for further dyeing.

As shown in Fig. 7b, K/S values of dyed fabrics were consistently high after 5 times reuse of the waste bath. The results demonstrated that Thy-Men from the spent bath had good reusability.

a

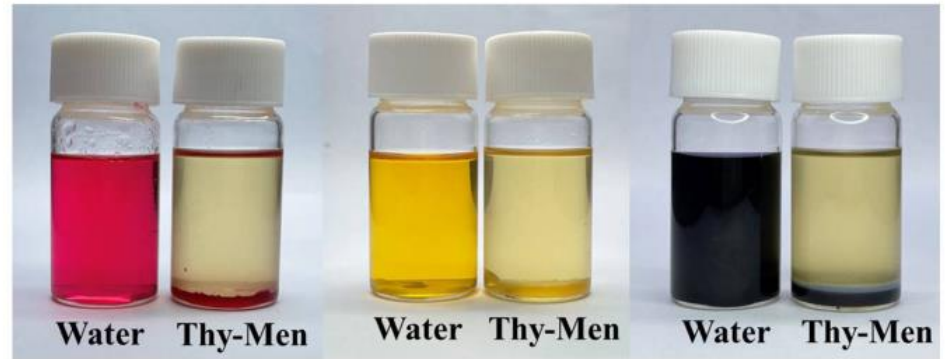

b

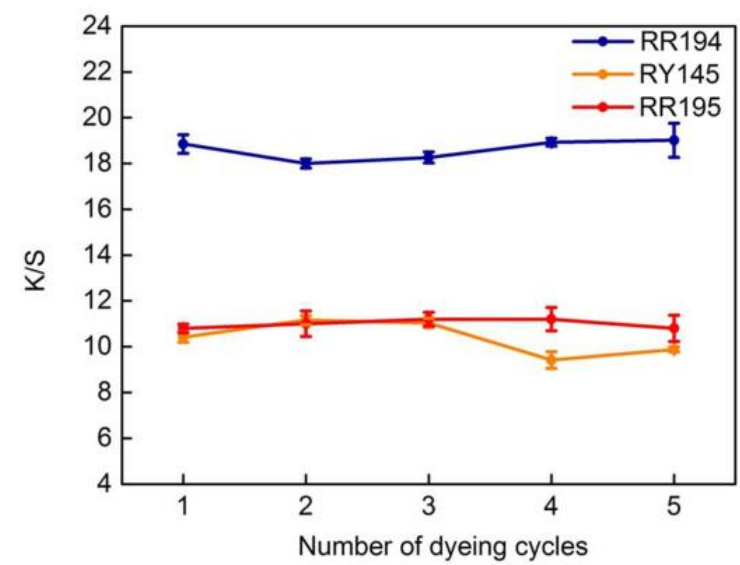

Fig. 7 (a) Dyeing effluent images for aqueous and Thy-Men dyeing of different dyes: RR195, RY145 and RB194, respectively; (b) K/S values after each dyeing cycle

\section{Dye fixation kinetics for aqueous and Thy-Men dyeing}

The fixation rate curves of Reactive Red 195 in different dyeing media were shown in Fig. 8a. The amount of fixation increased rapidly at the beginning of dyeing, the fixation rate slowed down gradually with the extension of time and the dyeing tends to be balanced. There was no obvious difference in the fixation rate of RR195 between the two dyeing systems. 

pseudo-first-order and pseudo-second-order dynamics models according to the formula (3) and (4).

$$
\begin{gathered}
\ln \left(\mathrm{q}_{\mathrm{e}}-\mathrm{q}_{\mathrm{t}}\right)=\ln \mathrm{q}_{\mathrm{e}}-\mathrm{k}_{1} \mathrm{t} \\
\frac{\mathrm{t}}{\mathrm{q}_{\mathrm{t}}}=\frac{1}{\mathrm{k}_{2} \mathrm{q}_{\mathrm{e}}^{2}}+\frac{\mathrm{t}}{\mathrm{q}_{\mathrm{e}}}
\end{gathered}
$$

where $q_{e}$ and $q_{t}(\mathrm{mg} / \mathrm{g})$ are the amounts of reactive dye on fibers at equilibrium and given time $\mathrm{t}$, respectively. $\mathrm{k}_{1}\left(\mathrm{~min}^{-1}\right)$ and $\mathrm{k}_{2}\left(\mathrm{~g} \mathrm{mg}^{-1} \min ^{-1}\right)$ refer to the rate constant of the pseudo-first-order and pseudo-second-order dynamics models. in equations (5) to (7).

$$
\begin{gathered}
\mathrm{q}_{\mathrm{e}, \mathrm{cal}}=\frac{1}{\mathrm{a}} \\
\mathrm{k}_{2}=\frac{1}{\mathrm{bq}_{\mathrm{e}}^{2}} \\
\mathrm{t}_{1 / 2}=\frac{1}{\mathrm{k}_{2} \mathrm{q}_{\mathrm{e}, \mathrm{cal}}}
\end{gathered}
$$

Where $\mathrm{q}_{\mathrm{e}, \mathrm{cal}}$ is the theoretical value of dyeing equilibrium, $\mathrm{k}_{2}$ is the dyeing rate constant, $t_{1 / 2}$ is half dyeing time, $a$ and $b$ are slope and intercept of pseudo-second-order kinetics linear fitting, $\mathrm{q}_{\mathrm{e}}$ is the actual dyeing equilibrium value. The fitted line plots of pseudo-first-order and pseudo-second-order dynamics models in conventional and Thy-Men dyeing systems are shown in Fig. 8b and Fig. 8c, respectively. Kinetic parameters of pseudo-second-order for fixation of Reactive Red 195 in conventional dyeing system and Thy-Men dyeing system are listed in Table 3. 
Both conventional dyeing system and Thy-Men dyeing system showed a better correlation coefficient value $(\mathrm{R})$ from the pseudo-second-order model than that from the pseudo-first-order model. Furthermore, the calculated $\mathrm{q}_{\mathrm{e}, \mathrm{cal}}$ values from the pseudo-second-order model fit well with the experimental adsorption capacities Red 195 on cotton fabrics in conventional dyeing system and Thy-Men dyeing system. $\mathrm{K}_{2}, \mathrm{t}_{1 / 2}$ listed in Table 3 are main factors in the fixation rate. The fixation rate of the Men-Thy system is similar to that of the conventional system, which ensures that the can obtain good leveling properties.
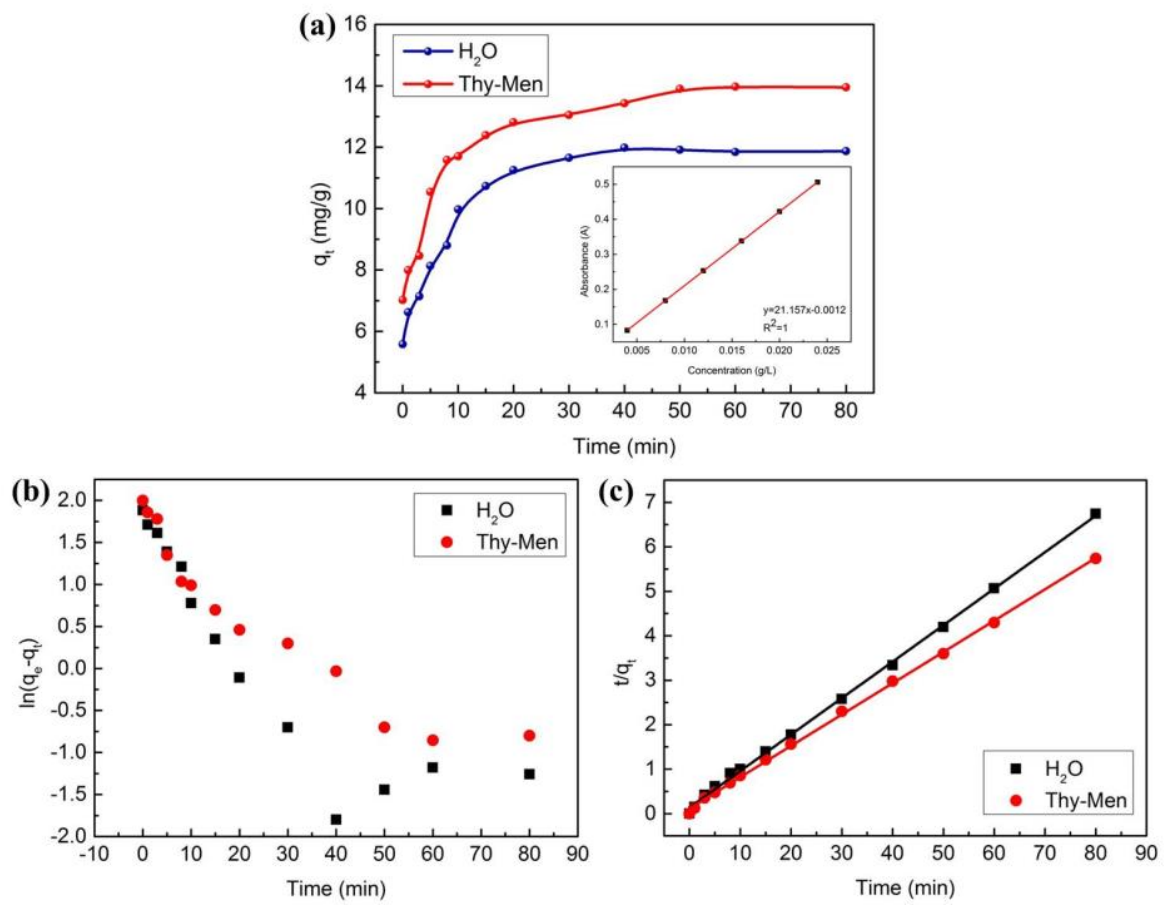

Fig. 8 (a) The fixation rate curve of Reactive Red 195. The fixation kinetics for the dyeing of cotton fabrics with Reactive Red 195: (b) $\ln \left(\mathrm{q}_{\mathrm{e}}-\mathrm{q}_{\mathrm{t}}\right)$ versus time; (c) t/ $\mathrm{q}_{\mathrm{t}}$ 
Table 3 Kinetic parameters of pseudo-second-order for fixation of Reactive Red 195 in aqueous dyeing system and Thy-Men dyeing system

\begin{tabular}{|c|c|c|c|c|c|}
\hline Dyeing system & $\begin{array}{c}\mathrm{k}_{2} \\
(\mathrm{~g} / \mathrm{mg} \cdot \min )\end{array}$ & $\begin{array}{l}\mathrm{q}_{\mathrm{e}, \exp } \\
(\mathrm{mg} / \mathrm{g})\end{array}$ & $\begin{array}{c}\mathrm{q}_{\mathrm{e}, \mathrm{cal}} \\
(\mathrm{mg} / \mathrm{g})\end{array}$ & $\begin{array}{l}\mathrm{t}_{1 / 2} \\
(\mathrm{~min})\end{array}$ & $\mathrm{R}^{2}$ \\
\hline $\begin{array}{l}\text { Conventional } \\
\text { aqueous system }\end{array}$ & 0.0494 & 12.152 & 12.195 & 1.66 & 0.9988 \\
\hline Thy-Men system & 0.0430 & 14.397 & 14.205 & 1.64 & 0.9991 \\
\hline
\end{tabular}

351

352

353

354

355

356

357

358

359

360

361

Influence of different dyeing systems on the physical properties of cotton fabrics

Fig. 9a shows the variation of tensile properties of cotton fabrics after dyeing with different systems. The tensile strength and elongation at break of cotton fabric dyed in system did not change significantly. The results showed that Thy-Men as dyeing medium did not affect the tensile properties of cotton fabric.

Fig. 9b shows the XRD patterns of cotton fabrics dyed in different systems.

Undyed cotton fabric, cotton fabric dyed in traditional system and cotton fabric dyed in Thy-Men system all had peak values at $14^{\circ} 、 16.7^{\circ}$ and $22.7^{\circ}$, and there was no significant difference in the intensity of the peak. The crystallinity was $66.9 \%, 68.7 \%$ and $64.4 \%$, respectively. In general, Thy-Men dyeing system did not affect the crystallinity of cotton fabrics. 

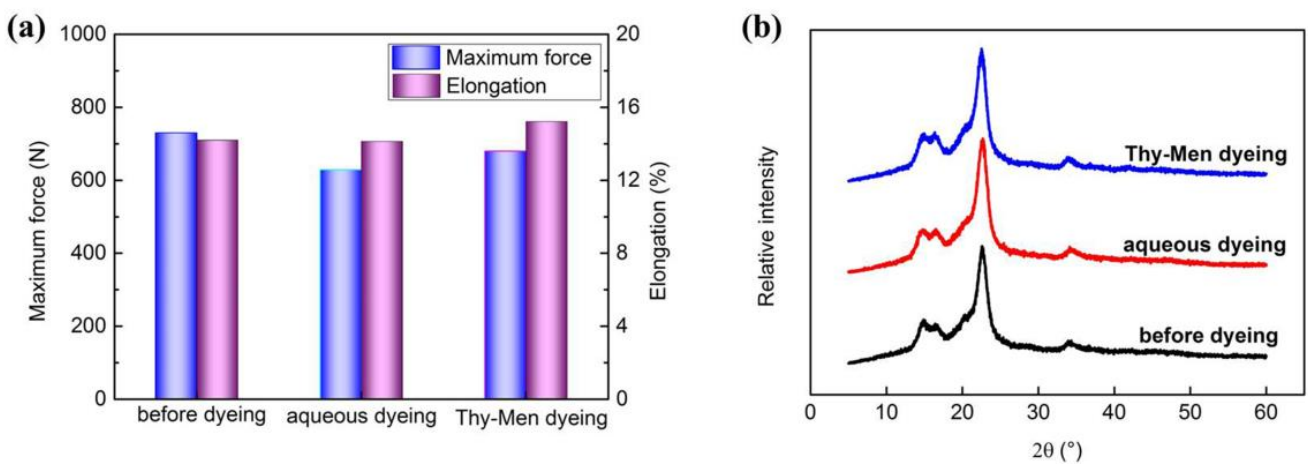

Fig. 9 (a) Tensile properties and (b) XRD spectra of cotton fabrics

\section{Evaluation of material costs}

The calculation of material costs is listed in Table 4. In Thy-Men dyeing system, more than $98 \%$ of the spent bath could be reused, so that the materials consumption of each dyeing cycle is low. In terms of material loss per cycle, the Thy-Men system is $\$ 0.036$ cheaper than the traditional dyeing system for dyeing $1 \mathrm{~kg}$ of cotton fabric. Although the initial cost of menthol and thymol is more expensive, the cost of wastewater treatment will be greatly reduced because of low emission from the Thy-Men system. With increasingly strict requirements of will gradually decrease with the increase of dyeing cycles. In summary, total cost of thymol-menthol dyeing system is unlikely to be higher than that of conventional dyeing system in the long term. 
fabric

\begin{tabular}{|c|c|c|c|c|c|c|c|c|}
\hline & \multicolumn{4}{|c|}{ Thy-Men system } & \multicolumn{4}{|c|}{ Conventional aqueous system } \\
\hline & \multirow{3}{*}{ Medium } & \multirow{3}{*}{ Dye } & \multirow{3}{*}{ Salt } & \multirow{3}{*}{ Alkali } & \multirow[t]{2}{*}{ Mediu } & \multirow{3}{*}{ Dye } & \multirow{3}{*}{ Salt } & \multirow{3}{*}{ Alkali } \\
\hline & & & & & & & & \\
\hline & & & & & $\mathrm{m}$ & & & \\
\hline & $0.010^{\mathrm{b}}$ (Menthol) & & & & & & & \\
\hline \multicolumn{9}{|l|}{ Consumptio } \\
\hline & $0.010^{\mathrm{b}}$ (Thymol) & & & & & & & \\
\hline \multirow[t]{3}{*}{$\mathrm{n}(\mathrm{kg})$} & & 0.02 & 0 & 0.06 & $10^{\mathrm{c}}$ & 0.02 & $0.4^{\mathrm{d}}$ & 0.2 \\
\hline & $1^{\mathrm{c}}$ (water) & & & & & & & \\
\hline & 9.28 (Menthol) & & & & & & & \\
\hline \multicolumn{9}{|l|}{ Unit price } \\
\hline & 1.55 (Thymol) & & & & & & & \\
\hline \multirow[t]{2}{*}{$(\$ / k g)^{\mathrm{a}}$} & & 11.6 & 0.26 & 0.26 & 0.0004 & 11.6 & 0.26 & 0.26 \\
\hline & 0.0004 (water) & & & & & & & \\
\hline \multirow[t]{2}{*}{ Material } & & 0.23 & & 0.015 & & 0.23 & 0.10 & \\
\hline & 0.1087 & & 0 & & 0.004 & & & 0.052 \\
\hline $\operatorname{cost}(\$)^{\mathrm{a}}$ & & 2 & & 6 & & 2 & 4 & \\
\hline
\end{tabular}

381 a All the prices of chemicals were obtained from Alibaba.com, accessed on March

$382 \quad 03,2021$.

$383 \quad{ }^{b}$ It is assumed that $2 \%$ Menthol - Thymol is lost after each cycle. To synthesize

$384 \quad 1 \mathrm{~kg}$ of Men-Thy hydrophobic deep eutectic solvent, $0.509 \mathrm{~kg}$ of menthol and

$385 \quad 0.490 \mathrm{~kg}$ of thymol are required.

$386 \quad{ }^{\mathrm{c}}$ Water content in Thy-Men system has the same weight as fabrics. The liquor

387 ratio is $20: 1$ in the conventional system. 
d $40 \mathrm{~g} / \mathrm{L}$ sodium sulfate is added to the conventional dyeing system.

389

\section{Conclusions}

In this research, a Thymol-Menthol hydrophobic deep eutectic solvent system was developed for reactive dyeing of cotton. The samples dyed from the developed dyeing system can achieve the same color strength as those from conventional dyeing system without salt and dispersing agents. Furthermore, the tone and chromaticity, colorfastness, as well as physical properties of the dyed cotton fabrics were not affected. Cross-sectional images of the cotton yarns illustrated that excellent permeability was also obtained. The color fixation behavior of cotton fabric in Thy-Men dyeing system has no significant difference from that in conventional system, which ensures satisfactory levelness. Excellent color consistency of the dyed fabric in a 5-cycle reuse sequence demonstrated that the spent bath had good reusability. Besides, considering water conservation and wastewater treatment, the cost of dyeing in Thy-Men system will not be too high from a long-term perspective.

Therefore, green reactive dyeing of cotton can be realized with the HDES system. It is of great significance to the sustainable development of the textile industry.

\section{Declarations}

\section{Acknowledgements}

This research was financially supported by the National Key R\&D Program of China (2017YFB0309200), Fundamental Research Funds for the Central Universities (JUSRP51717A) and International Joint Research Laboratory for Eco- Textile Technology at Jiangnan University. 


\section{Compliance with Ethical Standards}

Conflicts of interest The authors declare no competing financial interest.

This article does not contain any studies with human participants or animals performed by any of the authors.

Informed consent was obtained from all individual participants included in the study.

Ethical statement I certify that this manuscript is original and has not been published and will not be submitted elsewhere for publication while being considered by cellulose. And the study is not split up into several parts to increase the quantity of submissions and submitted to various journals or to one journal over time. No data have been fabricated or manipulated (including images) to support our conclusions.

No data, text, or theories by others are presented as if they were our own.

\section{References}

Anastas PT, Warner JC (1998) Green chemistry: Theory and practice. Abstracts of Papers of the American Chemical Society 244:19758-19771

Arivithamani N, Giri Dev VR (2017) Sustainable bulk scale cationization of cotton hosiery fabrics for salt-free reactive dyeing process. J Clean Prod 149:1188-1199

Ayadi I, Souissi Y, Jlassi I, Peixoto F, Mnif W (2016) Chemical synonyms, molecular structure and toxicological risk assessment of synthetic textile dyes: a critical review. J Dev Drugs 5:2

Cao J, Yang M, Cao F, Wang J, Su E (2017) Well-designed hydrophobic deep eutectic solvents as green and efficient media for the extraction of artemisinin from artemisia annua leaves. ACS Sustain Chem Eng 5:3270-3278

Chavan RB, Rao JV (1983) Dyeing of water swollen, solvent exchanged cotton with anionic dyes from dimethylformamide-trichloroethylene solvent mixtures. Tex Res J 53:290-297

Chen L, Wang B, Chen J, Ruan X, Yang Y (2015) Comprehensive study on cellulose swelling for completely recyclable nonaqueous reactive dyeing. Ind Eng Chem Res 54:2439-2446

Cid MF, Gerstner KN, Spronsen JV, Van D, Veugelers W, Woerlee GF, Witkamp GJ (2007) Novel process to enhance the dyeability of cotton in supercritical carbon dioxide. Text Res $\mathrm{J}$ 77:38-46

Dong W, Zhou M, Li Y, Zhai S, Jin K, Fan Z, Zhao H, Zou W, Cai Z (2020) Low-salt dyeing of cotton fabric grafted with ph-responsive cationic polymer of polyelectrolyte 2-(n,n-dimethylamino)ethyl methacrylate. Colloid Surface A 594:124573 
Durand E, Lecomte J, Villeneuve P (2016) From green chemistry to nature: The versatile role of low transition temperature mixtures. Biochimie 120:119-123

Fernandez Cid MV, Van Spronsen J, Van der Kraan M, Veugelers WJT, Woerlee GF, Witkamp GJ (2005) Excellent dye fixation on cotton dyed in supercritical carbon dioxide using fluorotriazine reactive dyes. Green Chem 7:609-616

Florindo C, Oliveira FS, Rebelo LPN, Fernandes AM, Marrucho IM (2014) Insights into the synthesis and properties of deep eutectic solvents based on cholinium chloride and carboxylic acids. ACS Sustain Chem Eng 2:2416-2425

Fu C, Wang J, Shao J, Pu D, Chen J, Liu J (2015) A non-aqueous dyeing process of reactive dye on cotton. J Text Inst 106:152-161

Hauser PJ, Tabba AH (2001) Improving the environmental and economic aspects of cotton dyeing using a cationised cotton. Color Technol

Jiang M, Ye K, Deng J, Lin J, Ye W, Zhao S, Van der Bruggen B (2018) Conventional ultrafiltration as effective strategy for dye/salt fractionation in textile wastewater treatment. Environ Sci Technol 52:10698-10708

Kissa E (1971) Lightfastness of reactive dyes. Text Res J 41:715-719

Li W, Mu B, Yang Y (2019) Feasibility of industrial-scale treatment of dye wastewater via bio-adsorption technology. Bioresour Technol 277:157-170

Libra JA, Borchert M, Vigelahn L, Storm T (2004) Two stage biological treatment of a diazo reactive textile dye and the fate of the dye metabolites. Chemosphere 56:167-180

Liu L, Mu B, Li W, Yang Y (2019) Semistable emulsion system based on spent cooking oil for pilot-scale reactive dyeing with minimal discharges. ACS Sustain Chem Eng 7:13698-13707

Long J-J, Xu H-M, Cui C-L, Wei X-C, Chen F, Cheng A-K (2014) A novel plant for fabric rope dyeing in supercritical carbon dioxide and its cleaner production. J Clean Prod 65:574-582

Luo X, White J, Thompson R, Rayner C, Kulik B, Kazlauciunas A, He W, Lin L (2018) Novel sustainable synthesis of dyes for clean dyeing of wool and cotton fibres in supercritical carbon dioxide. J Clean Prod 199:1-10

Ma W, Meng M, Yan S, Zhang S (2016) Salt-free reactive dyeing of betaine-modified cationic cotton fabrics with enhanced dye fixation. Chinese Journal of Chemical Engineering 24:175-179

Mu B, Li W, Xu H, Emanuel L, Yang Y (2019a) Salt-free and environment-friendly reactive dyeing of cotton in cottonseed oil/water system. Cellulose 26:6379-6391

Mu B, Liu L, Li W, Yang Y (2019b) High sorption of reactive dyes onto cotton controlled by chemical potential gradient for reduction of dyeing effluents. J Environ Manag 239:271-278

Mu B, Liu L, Li W, Yang Y (2019c) A water/cottonseed oil bath with controllable dye sorption for high dyeing quality and minimum discharges. J Clean Prod 236:117566

Paiva A, Craveiro R, Aroso I, Martins M, Reis RL, Duarte ARC (2014) Natural deep eutectic solvents - solvents for the 21st century. ACS Sustain Chem Eng 2:1063-1071

Pei L, Luo Y, Saleem MA, Wang J (2021) Sustainable pilot scale reactive dyeing based on silicone oil for improving dye fixation and reducing discharges. J Clean Prod 279:123831

Shu D, Fang K, Liu X, Cai Y, Zhang X, Zhang J (2018) Cleaner coloration of cotton fabric with reactive dyes using a pad-batch-steam dyeing process. J Clean Prod 196:935-942

Sparham C, Van Egmond R, O'Connor S, Hastie C, Whelan M, Kanda R, Franklin O (2008) Determination of decamethylcyclopentasiloxane in river water and final effluent by 
headspace gas chromatography/mass spectrometry. J Chromatogr A 1212:124-129

Suwanruji P, Freeman HS (2010) Design, synthesis and application of easy wash-off reactive dyes. Color Technol 122:27-36

Tam VWY, Tam CM (2006) A review on the viable technology for construction waste recycling. Resour Conserv and Recycl 47:209-221

Vakili M, Rafatullah M, Salamatinia B, Abdullah AZ, Ibrahim MH, Tan KB, Gholami Z, Amouzgar P (2014) Application of chitosan and its derivatives as adsorbents for dye removal from water and wastewater: A review. Carbohyd Polym 113:115-130

Van Osch DJ, Parmentier D, Dietz CH, van den Bruinhorst A, Tuinier R, Kroon MC (2016) Removal of alkali and transition metal ions from water with hydrophobic deep eutectic solvents. Chem Commun (Camb) 52:11987-11990

Van Osch DJGP, Dietz CHJT, van Spronsen J, Kroon MC, Gallucci F, van Sint Annaland M, Tuinier R (2019) A search for natural hydrophobic deep eutectic solvents based on natural components. ACS Sustain Chem Eng 7:2933-2942

Wang A, Xia L, Zhou S, Guo H, Wang Y, Xu W (2020) Solvent assisted reactive dyeing of cotton with high exhaustion in the absence of salt. J Clean Prod 267:122062

Wang L, Ma W, Zhang S, Teng X, Yang J (2009) Preparation of cationic cotton with two-bath pad-bake process and its application in salt-free dyeing. Carbohyd Polym 78:602-608

Xia L, Wang A, Wang Y, Zhang C, Wang Y, Zhou S, Fu Z, Zhao H, Ding C, Xu W (2021) Eco-friendly dyeing of raw cotton fibres in an ethanol-water mixture without scouring and bleaching pretreatments. Green Chem 23:796-807

Xia L, Wang A, Zhang C, Liu Y, Guo H, Ding C, Wang Y, Xu W (2018) Environmentally friendly dyeing of cotton in an ethanol-water mixture with excellent exhaustion. Green Chem 20:4473-4483

Xia L, Zhou S, Zhang C, Fu Z, Wang A, Zhang Q, Wang Y, Liu X, Wang X, Xu W (2020) Environment-friendly juncus effusus-based adsorbent with a three-dimensional network structure for highly efficient removal of dyes from wastewater. J Clean Prod 259:120812

Zhao J, Agaba A, Sui X, Mao Z, Xu H, Zhong Y, Zhang L, Wang B (2018) A heterogeneous binary solvent system for recyclable reactive dyeing of cotton fabrics. Cellulose 25:7381-7392 
Figures

(a)<smiles></smiles>

(b)<smiles>NC(=O)Nc1cc(Nc2nc(Cl)nc(Nc3cccc(OC(=O)CO[N+](=O)[O-])c3)n2)ccc1N=Nc1cc2c([N+](=O)[O-])cc(OS(=O)(=O)[O-])cc2cc1[N+](=O)[O-]</smiles>

(c)<smiles></smiles>

Figure 1

Chemical structure of (a) C. I. Reactive Red 195; (b) C. I. Reactive Yellow 145; (c) C. I. Reactive Blue 194 
(a)
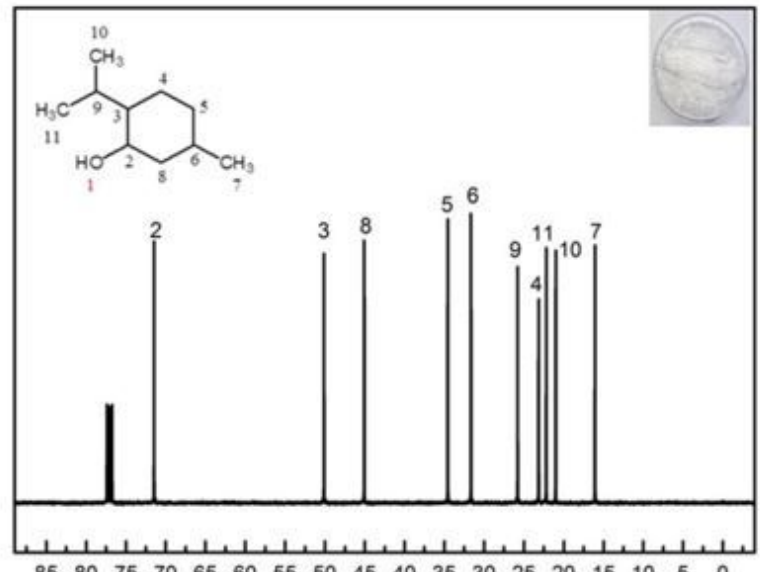

$85807570 \quad 65 \quad 60 \quad 55 \quad 50 \quad 45 \quad 40 \quad 35 \quad 30 \quad 25 \quad 20 \quad 15 \quad 10 \quad 5 \quad 0$ $\mathrm{f} 1$ (ppm)

(c)

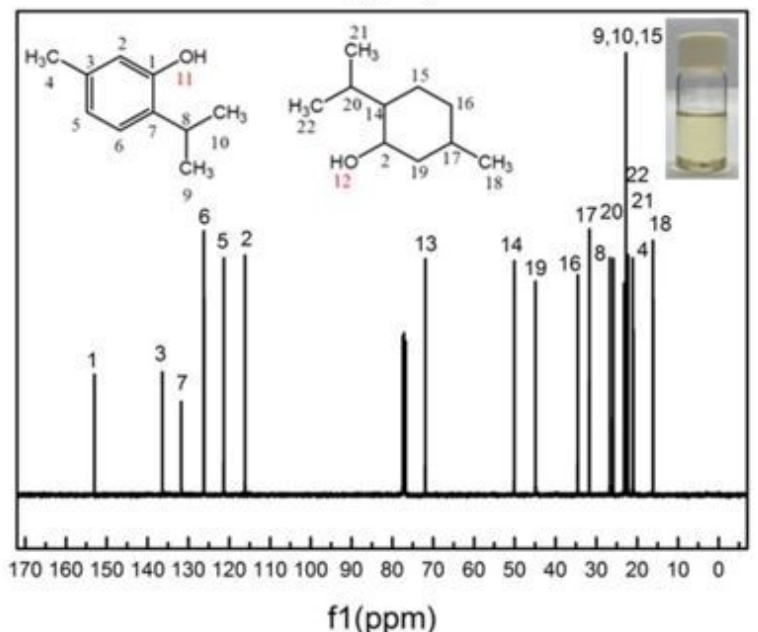

(b)

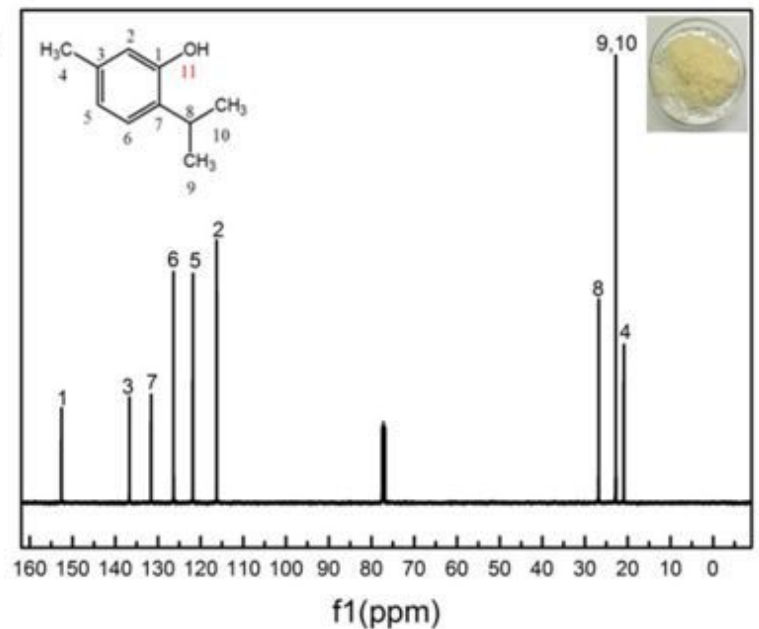

Figure 2

13C NMR of (a) menthol (b) thymol (c) Thy: Men (1:1). Insets show photos of menthol, thymol and Thy: Men, respectively 

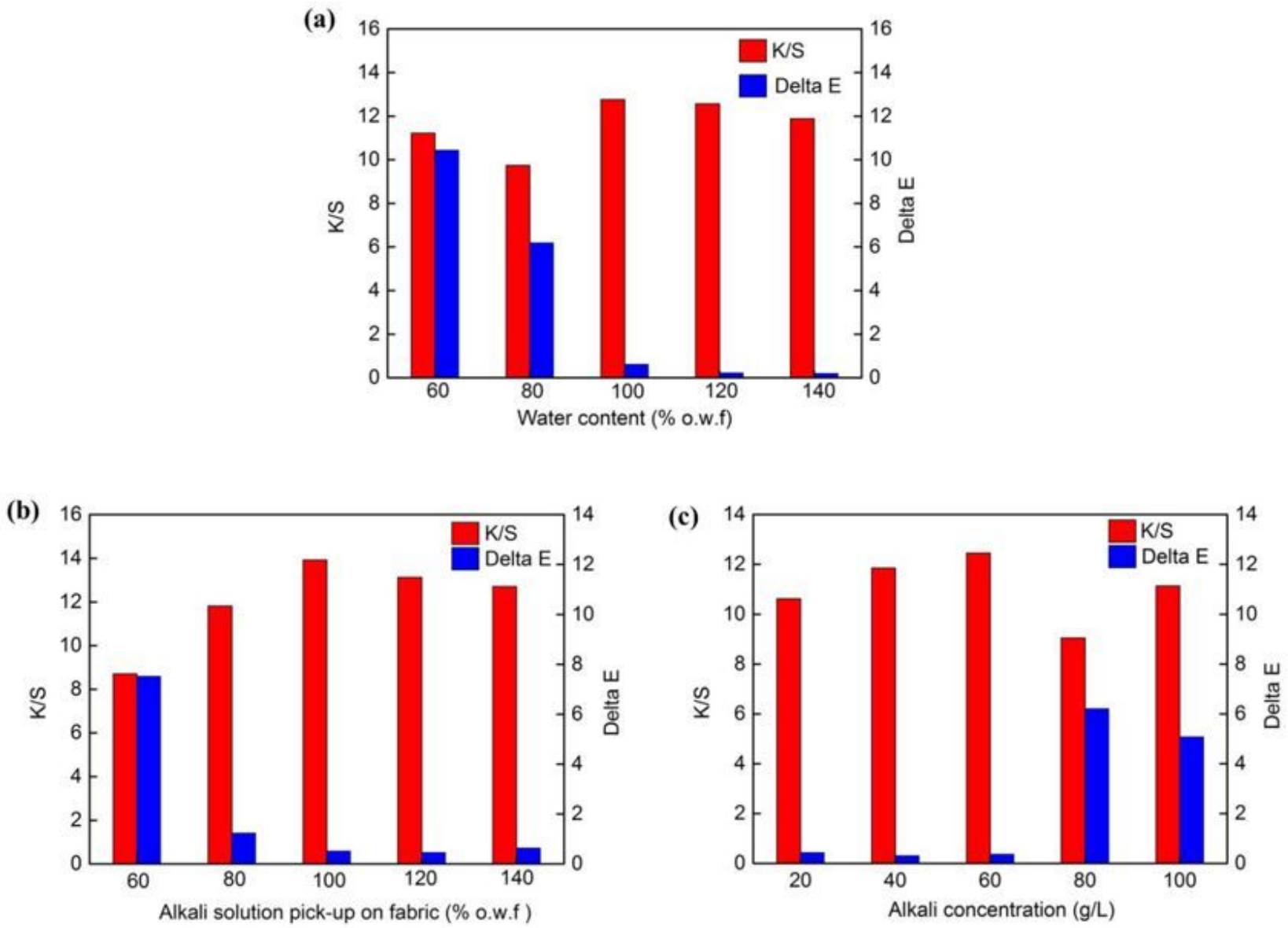

Figure 3

Effect of (a) water content (b) pick-up and (c) concentration of alkali solution on color strength (K/S) and levelness $(\Delta \mathrm{E})$ 


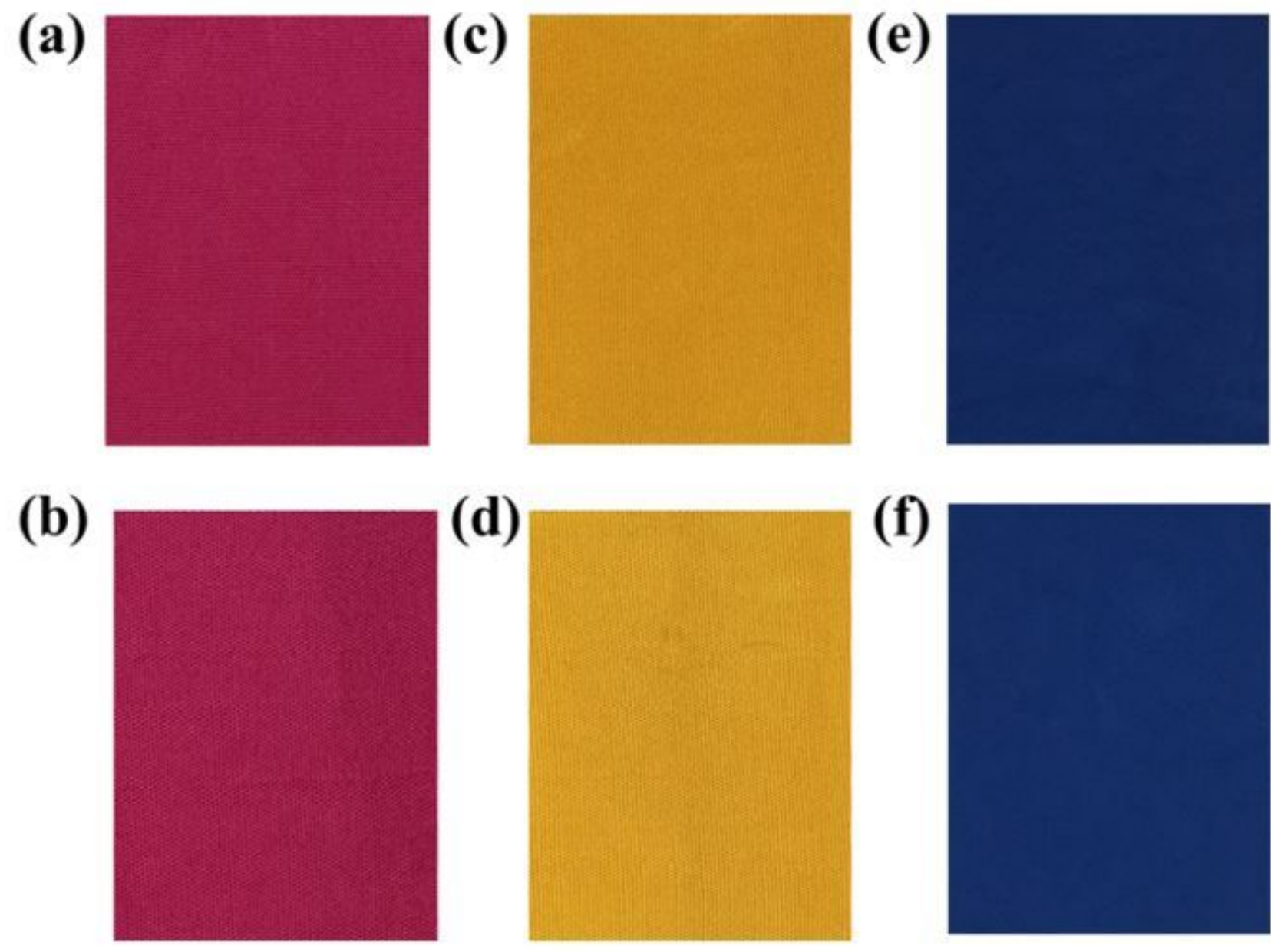

Figure 4

Photos of fabrics dyed with: (a) RR195 (c) RY145 (e) RB194 in aqueous system; (b) RR195 (d) RY145 (f) RB194 in the Thy-Men system 

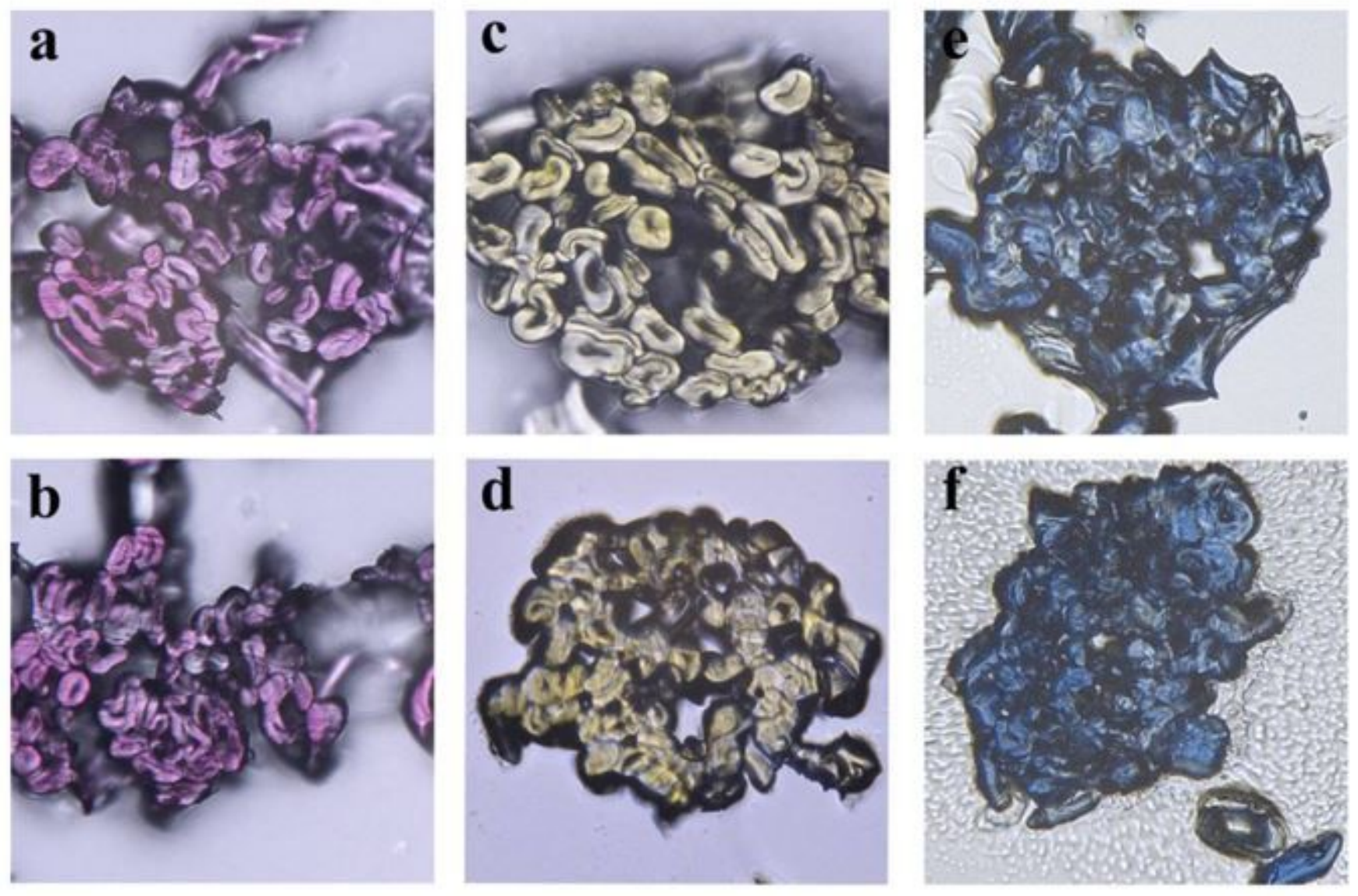

\section{Figure 5}

Cross-sectional images of cotton yarns dyed with: (a) RR195 (c) RY145 (e) RB194 in aqueous system; (b) RR195 (d) RY145 (f) RB194 in the Thy-Men system 
(a)

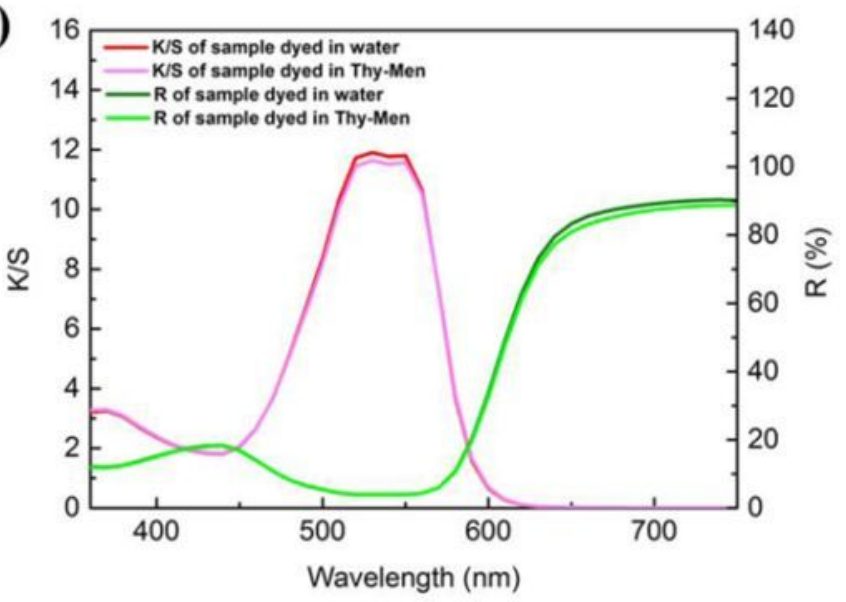

(c)

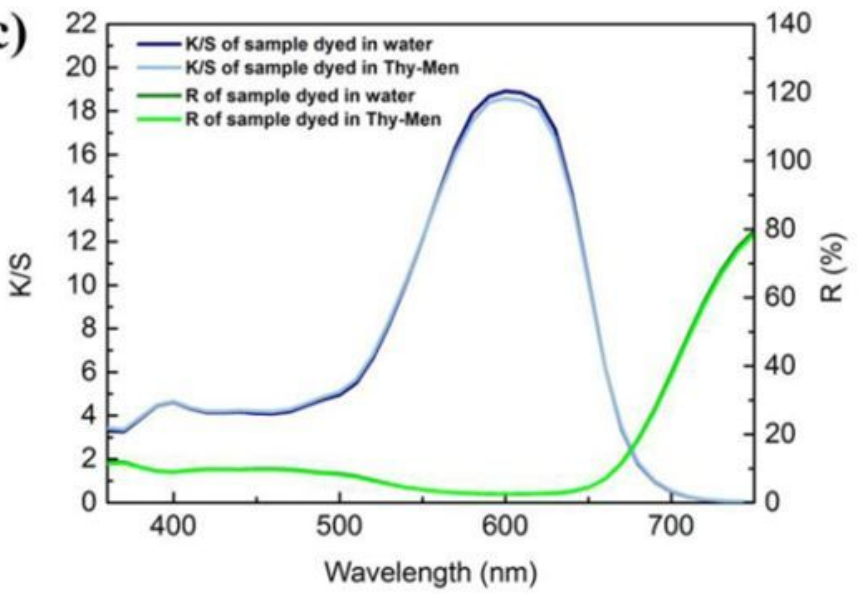

(b)

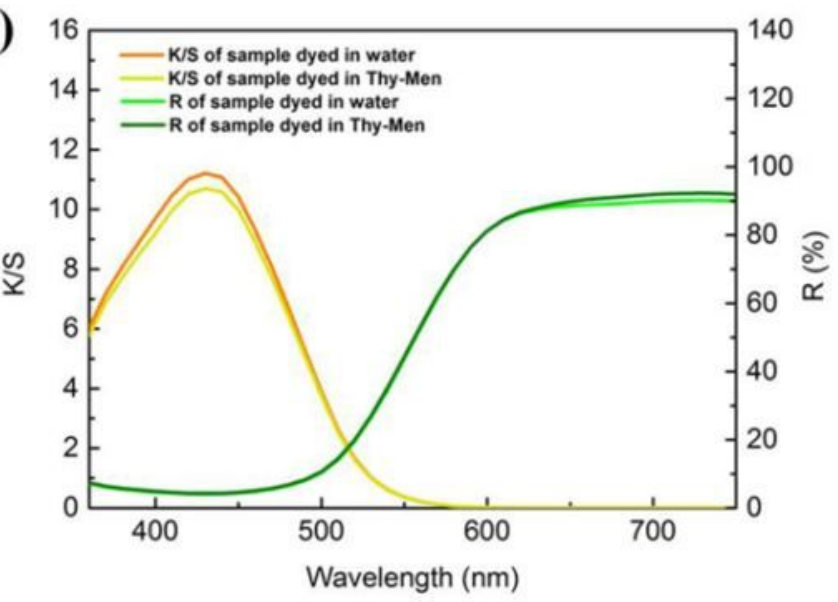

Figure 6

Reflectance (R) and K/S curves of fabrics dyed with: (a) RR195; (b) RY145; (c) RB194 in the Thy-Men system 


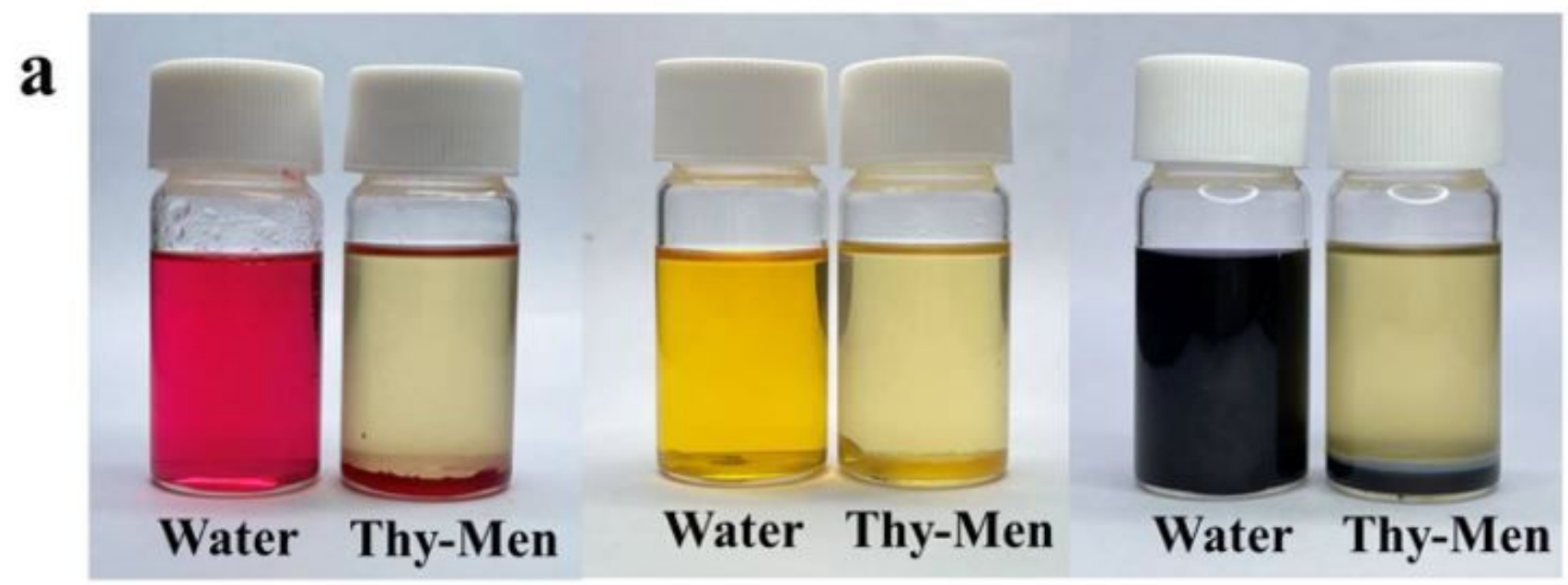

b

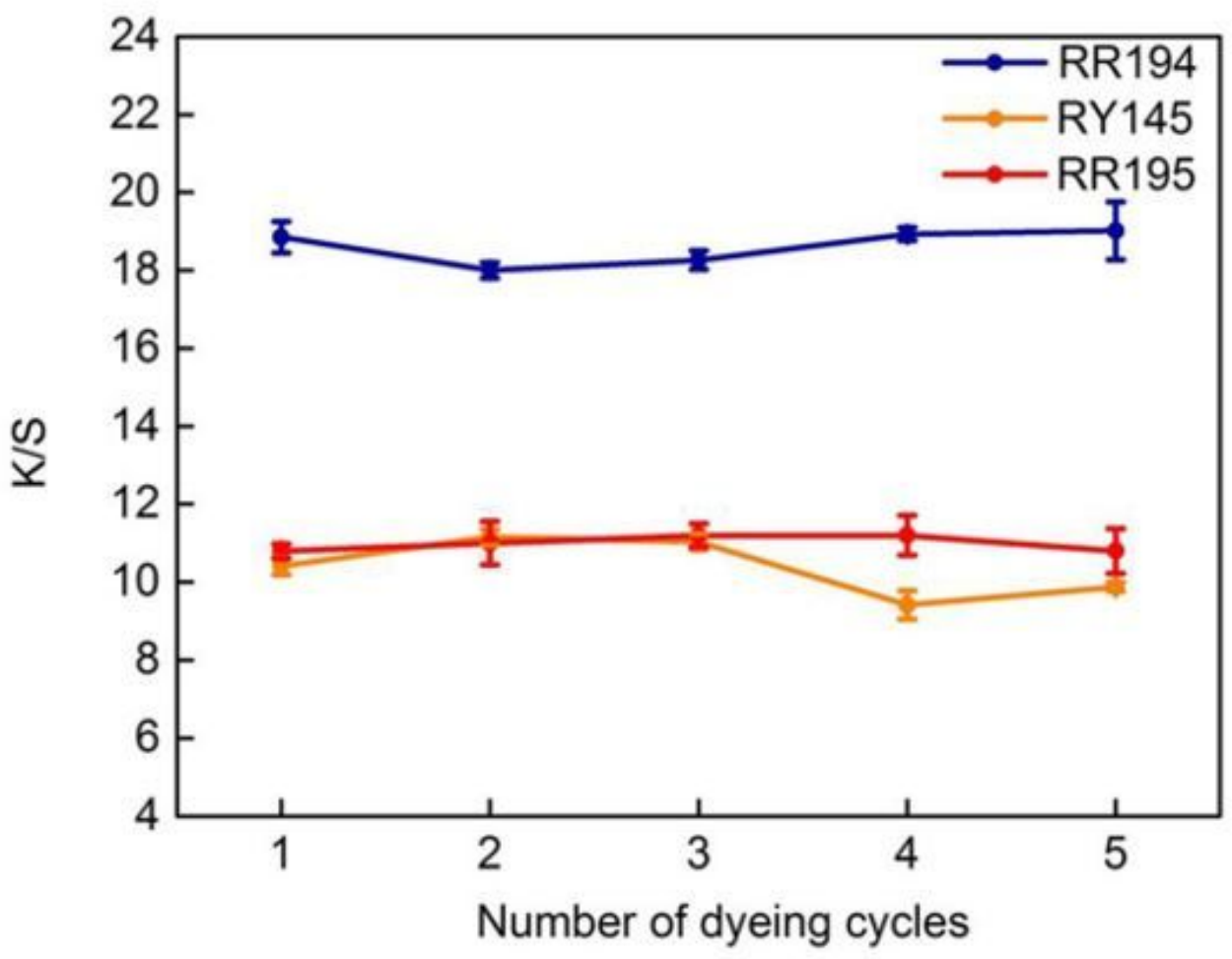

Figure 7

(a) Dyeing effluent images for aqueous and Thy-Men dyeing of different dyes: RR195, RY145 and RB194, respectively; (b) K/S values after each dyeing cycle 

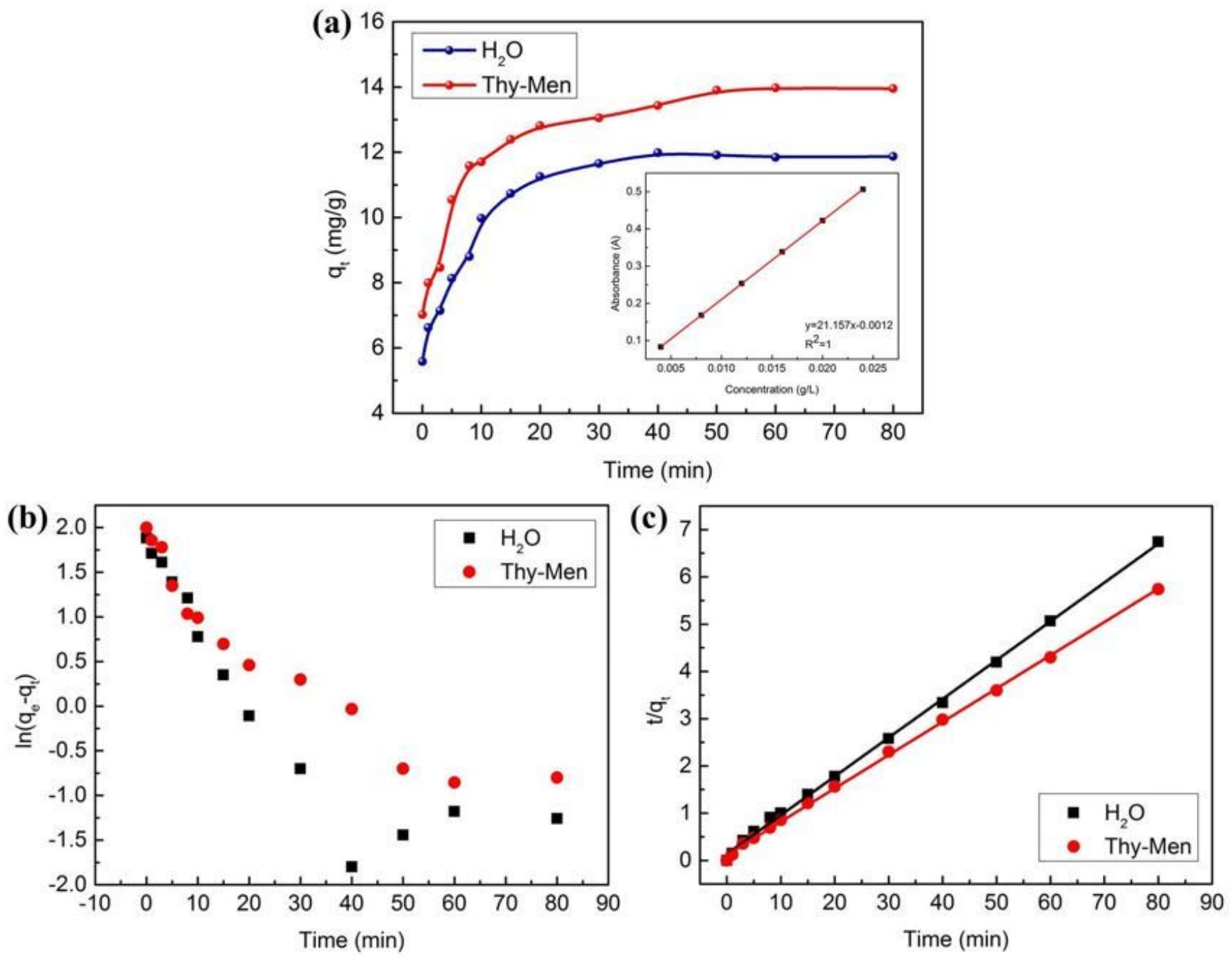

Figure 8

(a) The fixation rate curve of Reactive Red 195. The fixation kinetics for the dyeing of cotton fabrics with Reactive Red 195: (b) In(qe - qt) versus time; (c) t/qt versus time 

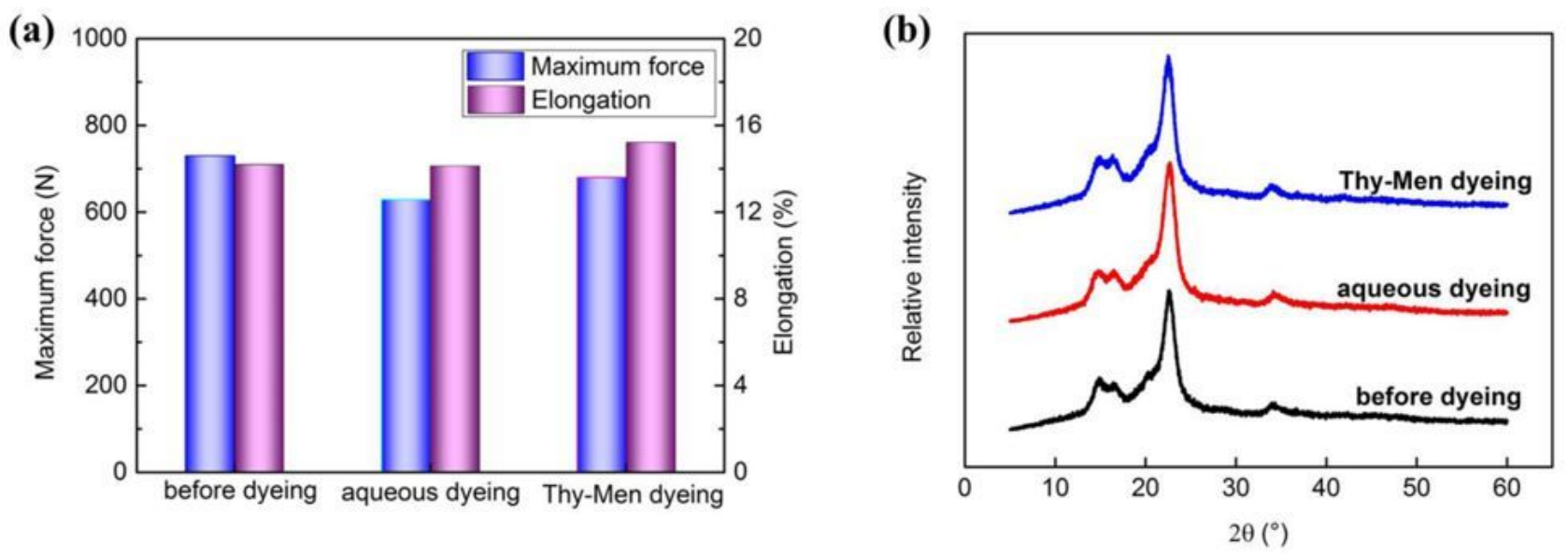

Figure 9

(a) Tensile properties and (b) XRD spectra of cotton fabrics

\section{Supplementary Files}

This is a list of supplementary files associated with this preprint. Click to download.

- scheme1.jpg

- Graphicabstract.docx 